\title{
On Analytic Multivalent Function Associated with Cosine Hyperbolic Function
}

\author{
Azam Muhammad ${ }^{1}$
}

1. School of Mathematics, Faculty of Applied science, Beijing University of Technology, Beijing, 100124, China, mazam18@yahoo.com,

Abstract: In this article, we set up some adequate conditions for analytic functions associated with cosine hyperbolic function. We determine conditions on $\alpha$ such that

$$
1+\alpha \frac{\mathcal{X}^{2+\mathcal{P}(j-1)} g^{\prime}(\mathcal{X})}{\mathcal{P} g^{j}(\mathcal{X})}, \text { for each } j=0,1,2,3
$$

are subordinated by Janowski function, then $\frac{g(x)}{\mathcal{x}^{\mathcal{P}}}<\cos \hbar(x),(x \in \mathfrak{C})$. By choosing specific values of functions $g$ we get some adequate conditions for multivalent starlike function related with cosine hyperbolic.

Keywords: Multivalent functions, Janowski function, Trigonometric functions

\section{Introduction and Definations}

To understand in a clear way the notions used in our results, we need to add here some basic literature of Geometric function theory. For this we start first with the notation $\mathcal{A}$ which denotes the class of holomorphic or analytic functions in the region $\mathbb{C}=\{\varkappa \in \mathbb{C}:|\varkappa|<1\}$ and if a function $g \in \AA$, then the relations $g(0)=g^{\prime}(0)-1=0$ must hold. Also, all univalent functions will be in a subfamily $\mathcal{S}$ of $\AA$. Furthermore to done the possibility of subjections between analytic functions $g_{1}$ and $g_{2}$, indicated by $g_{1}(x)<g_{2}(x)$, as; the functions $g_{1}, g_{2} \in \AA$, are associated by the connection of subjection, if there exists an analytic function $v$ with the limitations $v(0)=0$ and $|v(x)|<1$ such that $g_{1}(x)=g_{2}(v(x)$ ). Moreover, if the function $g_{2} \in \mathcal{S}$ in $\mathfrak{C}$, then we obtain.

$$
g_{1}(x)<g_{2}(x) \Leftrightarrow\left\{g_{1}(0)=g_{2}(0) \& g_{1}(\mathfrak{C}) \subset g_{2}(\mathfrak{C})\right\}
$$

In 1992, Ma and Minda [16] considered a holomorphic function $\psi(\mathcal{X})$ normalized by the conditions $\psi(0)=1$ and $\psi^{\prime}(0)>0$ with $\mathcal{R e} \psi>$ in $\mathfrak{C}$. The function $\psi$ maps the disc $\mathfrak{C}$ onto the region which is star-shaped about 1 and symmetric along the real axis.

In particular, the function $\psi(\mathcal{X})=(1+\AA \mathcal{X}) /(1+\mathcal{B} \mathcal{X}),(-1 \leq \mathcal{B}<\AA \leq 1)$ maps $\mathbb{C}$ onto the disc on the right half plane with center on the real axis and

diameter end points $\frac{1-\mathcal{F}}{1-\mathcal{G}}$ and $\frac{1+\mathcal{F}_{\varkappa}}{1+\mathcal{G}_{\varkappa}}$. This interesting familiar function is named as Janowski function [10]. The image of the function $\psi(x)=\operatorname{cash}(x)$ shows that the picture space is limited by the right half of the cosine hyperbolic [25]. The function $\psi(x)=1+\frac{4}{3} \mathcal{x}+\frac{2}{3} \mathcal{x}^{2}$ maps $\mathfrak{C}$ into the image set bounded by the cardioid given by $\left(9 d^{2}+9 e^{2}-8 e+5\right)^{2}-16\left(9^{2}+9 e^{2}-6 e+1\right)=0$, [21] in more studied in [23]. the function $\psi(\mathcal{X})=1+\sin \mathcal{X}$ was examined by Cho and his coauthors in [3] while $\psi(\mathcal{X})=\mathrm{e}^{\mathcal{X}}$ is of 
late studied in [17] and [24]. Further, by choosing particular $\psi$, several subclasses of starlike functions have been studied. See the details in $[2,4,5,11,12,14,19]$. Recently, Ali et al. [1] have acquired adequate conditions on $\alpha$ such that

$$
1+x g^{\prime}(x) / g^{i}(x)<\sqrt{1+x} \Rightarrow g(x)<\sqrt{1+x} \text {, for } i=0,1,2 .
$$

Comparative sort suggestions have been examined in a portion of the new papers by various analysts, for instance see the papers

contributed by Halim and Omar [6] Haq et al [7], Kumar et. al [13, 15] Paprocki and Sokol [18], Raza et al [20] and Sharma et al [22].

In 1994, Hayman [8] studied multivalent ( $\mathcal{P}$-valent ) functions which is a generalization of univalent functions and is defined as; an analytic function $g$ in an arbitrary domain $\mathbb{C} \subset \mathbb{C}$ is said to be $\mathcal{P}$-valent, if for every complex number $\omega$, the equation $g(x)=\omega$ has maximum $\mathcal{P}$ roots in $\mathfrak{C}$ and for a complex number $\omega_{0}$ the equation $g(\mathcal{X})=\omega_{0}$ has exactly $\mathcal{P}$ roots in $\mathfrak{C}$. Let $\AA_{\mathcal{P}}(\mathcal{P} \in \mathbb{N}=\{1,2, \ldots \ldots\})$ denote the class of functions, say $g \in \AA_{\mathcal{P}}$, that are multivalent holomorphic in the unit disc $\mathfrak{C}$ and which have the following series expansion:

$$
\mathcal{S}_{\mathrm{L}_{\mathcal{P}}}^{*}=\mathcal{H}^{\mathcal{P}}+\sum_{\mathrm{k}=\mathcal{P}+1}^{\infty} \mathrm{a}^{\mathrm{k}} \mathcal{H}^{\mathrm{k}}, \quad(\mathcal{x \in C})
$$

Using the idea of multivalent functions, we now introduce the class $\mathrm{S}_{\mathrm{L}_{\mathcal{P}}}^{*}$ of multivalent starlike functions associated with cosine hyperbolic function and as given below:

$$
\mathcal{S}_{\mathrm{L}_{\mathcal{P}}}^{*}=\left\{g(x) \in \AA_{\mathcal{P}}: \frac{x g^{\prime}(x)}{\mathcal{P} g(x)}<\operatorname{cas\hbar }(x),(x \in \mathfrak{C})\right\}
$$

In this paper, we determine conditions on $\alpha$ such that for each

$$
1+\alpha \frac{\mathcal{X}^{2+\mathcal{P}(j-1)} \mathcal{g}^{\prime}(\mathcal{X})}{\mathcal{P} g^{j}(\mathcal{X})}, \text { for each } j=0,1,2,3
$$

are subordinated to Janowski functions implies $\frac{g(x)}{\mathcal{X}^{\mathcal{P}}}<\cos \hbar(x),(\mathcal{x} \in \mathfrak{C})$, these results are utilized to show that $g$ are in the class $\mathcal{S}_{\mathrm{L}_{\mathcal{P}}}^{*}$.

Lemma 1.1. [9] Let $v$ be a non-constant analytic function in $\mathfrak{C}$ with $v(0)=0$. If

$$
\left|v\left(\mathcal{x}_{0}\right)\right|=\max \left\{|v(x)|,|x| \leq\left|\mathcal{x}_{0}\right|\right\}, x \in \mathbb{C}
$$

then there exists a real number $\mathrm{m}(\mathrm{m} \geq 1)$ such that $\varkappa_{0} \mathrm{v}^{\prime}\left(\varkappa_{0}\right)=\operatorname{lv}\left(\varkappa_{0}\right)$.

To evade redundancies, we accept that the accompanying limitations

$$
-1 \leq \mathcal{B}<\AA \leq 1 . j \in \mathbb{N}=\{1,2, \ldots . .\}, \mathrm{k} \in \mathbb{N}_{0}=\mathbb{N} \cup\{0\}
$$

otherwise we will state it where different. 


\section{Adequate Conditions Associated with Cosh}

Theorem 2.1. Let $g \in \AA_{\mathcal{P}}$ and satisfying

$$
1+\frac{\alpha \varkappa^{1-\mathcal{P}} g^{\prime}(\varkappa)}{\mathcal{P}}<\frac{1+\mathcal{F}_{\varkappa}}{1+\mathcal{G}_{\varkappa}}
$$

with the restriction on $\alpha$ is

$$
|\alpha| \geq \frac{(\mathcal{F}-\mathcal{G}) \mathcal{P}}{(\sin (1)-|\mathrm{B}| \sin \hbar(1))-(1+|\mathrm{B}| \mathcal{P} \cos \hbar(1))}
$$

Then

$$
\frac{g(x)}{\varkappa^{\mathcal{P}}}<\cos \hbar(x)
$$

Proof. Let us consider that

$$
\hbar(\mathcal{X})=1+\frac{\alpha \mathcal{\varkappa}^{1-\mathcal{P}} g^{\prime}(\mathcal{x})}{\mathcal{P}}
$$

Then the function $\hbar$ is holomorphic in $\mathfrak{C}$ with $\hbar(0)=1$. Additionally, suppose that

$$
v(x)=\left(\cos \hbar^{-1}\left(\frac{g(x)}{\mathcal{P}^{x}}\right)\right)
$$

Where we chose the rule parts of the function that are logarithmic and square root. At that point $\mathrm{v}$ is obviously a holomorphic function in $\mathfrak{C}$ with $v(0)=0$. likewise, since

$$
\cos \hbar^{-1}(\mathcal{x})=\ln \left[\varkappa+\sqrt{\mathcal{X}^{2}-1}\right]
$$

To finish the evidence of this outcome, we simply need to verification $|v(\mathcal{x})|<1$ in $\mathfrak{C}$. By virtueof $(2: 4)$, we have

$$
\hbar(x)=1+\frac{\alpha \varkappa v^{\prime}(x) \sin \hbar v(x)}{\mathcal{P}}+\alpha \cos \hbar v(x)
$$

Therefore

$$
\begin{aligned}
\left|\frac{\hbar(x)-1}{\mathcal{F}-\mathcal{G} \hbar(x)}\right| & =\left|\frac{\frac{\alpha \varkappa v^{\prime}(x) \sin \hbar v(x)}{\mathcal{P}}+\alpha \cos \hbar v(x)}{\mathcal{F}-\mathcal{G}\left(1+\frac{\alpha \varkappa v^{\prime}(x) \sin \hbar v(x)}{\mathcal{P}}+\alpha \cos \hbar v(x)\right)}\right| \\
& =\left|\frac{\alpha\left\{x v^{\prime}(x) \sin \hbar v(x)+\cos \hbar v(x)\right\}}{(\mathcal{F}-\mathcal{G}) \mathcal{P}-\operatorname{B} \alpha\left(x v^{\prime}(x) \sin \hbar v(x)+\mathcal{P} \cos \hbar v(x)\right)}\right|
\end{aligned}
$$

Presently we guess that a point $\mathcal{H}_{0} \in \mathfrak{C}$ happens with the end goal that

$$
\max _{|x| \leq\left|\mathcal{x}_{0}\right|} v(x)=\left|v\left(x_{0}\right)\right|=1
$$


Additionally, by Lemma (1.1), a number $\mathrm{m} \geq 1$, be alive with $\varkappa_{0} \mathrm{v}^{\prime}\left(\mathcal{x}_{0}\right)=\mathrm{mv}\left(\mathcal{H}_{0}\right)$. Furthermore, we likewise assume that $\mathrm{v}\left(\mathcal{x}_{0}\right)=\mathrm{e}^{\mathrm{i} \theta}$ for $\theta \in[-\pi, \pi]$. Then we have

$$
\begin{aligned}
& \left|\frac{\hbar\left(\varkappa_{0}\right)-1}{\mathcal{F}-\mathcal{G} \hbar\left(\varkappa_{0}\right)}\right|=\left|\frac{\alpha\left(\varkappa_{0} v^{\prime}\left(\varkappa_{0}\right) \sin \hbar v\left(\varkappa_{0}\right)+\mathcal{P} \operatorname{cas} \hbar v\left(\varkappa_{0}\right)\right)}{(\mathcal{F}-\mathcal{G}) \mathcal{P}-\alpha \mathcal{G}\left(\varkappa_{0} v^{\prime}\left(\varkappa_{0}\right) \sin \hbar v\left(\varkappa_{0}\right)+\mathcal{P} \cos \hbar v\left(\varkappa_{0}\right)\right)}\right| \\
& =\left|\frac{\alpha\left(\mathrm{me}^{\mathrm{i} \theta} \sin \hbar\left(\mathrm{e}^{\mathrm{i} \theta}\right)+\mathcal{P} \cos \hbar\left(\mathrm{e}^{\mathrm{i} \theta}\right)\right)}{(\mathcal{F}-\mathcal{G}) \mathcal{P}-\alpha \mathcal{G}\left(\mathrm{m}\left(\mathrm{e}^{\mathrm{i} \theta}\right) \sin \hbar\left(\mathrm{e}^{\mathrm{i} \theta}\right)+\mathcal{P} \cos \hbar\left(\mathrm{e}^{\mathrm{i} \theta}\right)\right)}\right| \\
& =\left|\frac{\alpha\left(\mathrm{m} \sin \hbar\left(\mathrm{e}^{\mathrm{i} \theta}\right)+\mathcal{P} \cos \hbar\left(\mathrm{e}^{\mathrm{i} \theta}\right)\right)}{(\mathcal{F}-\mathcal{G}) \mathcal{P}-\alpha \mathcal{G}\left(\mathrm{m} \sin \hbar\left(\mathrm{e}^{\mathrm{i} \theta}\right)+\mathcal{P} \cos \hbar\left(\mathrm{e}^{\mathrm{i} \theta}\right)\right)}\right| \\
& \geq \frac{|\alpha|\left(\mathrm{m}\left|\sin \hbar\left(\mathrm{e}^{\mathrm{i} \theta}\right)\right|-\mathcal{P}\left|\operatorname{cas} \hbar\left(\mathrm{e}^{\mathrm{i} \theta}\right)\right|\right)}{(\mathcal{F}-\mathcal{G}) \mathcal{P}+|\alpha||\mathcal{G}|\left(\mathrm{m}\left|\sin \hbar\left(\mathrm{e}^{\mathrm{i} \theta}\right)\right|+\mathcal{P}\left|\cos \hbar\left(\mathrm{e}^{\mathrm{i} \theta}\right)\right|\right)}
\end{aligned}
$$

If $|\mathcal{X}|=r,-\pi \leq \theta \leq \pi$, at that point basic estimation delineates that

$$
\begin{aligned}
& \left|\cos \hbar\left(\mathrm{e}^{\mathrm{i} \theta}\right)\right|^{2}=\cos \hbar^{2}(\cos \theta) \operatorname{cas}^{2}(\sin \theta)+\sin ^{2}(\cos \theta) \sin ^{2}(\sin \theta)=\delta(\theta) \\
& \left|\sin \hbar\left(\mathrm{e}^{\mathrm{i} \theta}\right)\right|^{2}=\sin \hbar^{2}(\cos \theta) \operatorname{cas}^{2}(\sin \theta)+\cos \hbar^{2}(\cos \theta) \sin ^{2}(\sin \theta)=\psi(\theta)
\end{aligned}
$$

A standard disentanglement guarantees that $0, \pm \pi, \frac{\pi}{2}$ are the roots of $\delta^{\prime}(\theta)=0$ and $\psi^{\prime}(\theta)=0$ in $[-\pi, \pi]$. Additionally, since

$$
\begin{aligned}
& \delta(\theta)=\delta^{\prime}(-\theta) \\
& \psi(\theta)=\psi^{\prime}(-\theta)
\end{aligned}
$$

it is sufficient to reason that $\theta \in[0, \pi]$ and along these lines we accomplish

$$
\begin{gathered}
\max \{\delta(\theta)\}=\delta(0)=\delta(\pi)=\cos \hbar^{2}(1) \\
\min \{\delta(\theta)\}=\delta\left(\frac{\pi}{2}\right)=\cos ^{2}(1) \\
\max \{\psi(\theta)\}=\psi(0)=\psi(\pi)=\sin \hbar^{2}(1) \\
\min \{\psi(\theta)\}=\psi\left(\frac{\pi}{2}\right)=\sin ^{2}(1)
\end{gathered}
$$

Thus, we have

$$
\begin{aligned}
& \cos (1) \leq\left|\cos \hbar\left(\mathrm{e}^{\mathrm{i} \theta}\right)\right| \leq \cos (1) \\
& \sin (1) \leq\left|\sin \hbar\left(\mathrm{e}^{\mathrm{i} \theta}\right)\right| \leq \sin (1)
\end{aligned}
$$

Therefore, using (2.6), (2.7), (2.5), we attain

$$
\left|\frac{\hbar\left(\varkappa_{0}\right)-1}{\mathcal{F}-\mathcal{G} \hbar\left(\varkappa_{0}\right)}\right| \geq \frac{|\alpha|(\operatorname{msin}(1)-\mathcal{P} \cos \hbar(1))}{(\mathcal{F}-\mathcal{G}) \mathcal{P}+|\alpha||\mathcal{G}|(\operatorname{msin} \hbar(1)+\mathcal{P} \cos \hbar(1))}
$$

Now, let 


$$
\Xi(\mathrm{m})=\frac{|\alpha|(m \sin (1)-\mathcal{P} \cos \hbar(1))}{(\mathcal{F}-\mathcal{G}) \mathcal{P}+|\alpha||\mathcal{G}|(m \sin \hbar(1)+\mathcal{P} \cos \hbar(1))}
$$

Then,

$$
\Xi^{\prime}(\mathrm{m})=\frac{|\alpha|(\mathcal{F}-\mathcal{G}) \mathcal{P} \sin (1)+|\alpha|^{2}|\mathcal{G}| \mathcal{P} \cos (1)(\sin (1)+\sin \hbar(1))}{(\mathcal{F}-\mathcal{G}) \mathcal{P}+|\alpha||\mathcal{G}|\{\operatorname{msin}(1)+\mathcal{P} \cos \hbar(1)\}^{2}}>0
$$

This conforms, that the function $\Xi$ is increasing and therefore $\Xi(m) \geq \Xi(1)$ for $m \geq 1$, so

$$
\left|\frac{\hbar\left(\varkappa_{0}\right)-1}{\mathcal{F}-\mathcal{G} \hbar\left(\varkappa_{0}\right)}\right| \geq \frac{|\alpha|(\sin (1)-\mathcal{P} \cos \hbar(1))}{(\mathcal{F}-\mathcal{G}) \mathcal{P}+|\alpha||\mathcal{G}|(\sin \hbar(1)+\mathcal{P} \cos \hbar(1))}
$$

Now, utilizing (2.2), we obtain

$$
\left|\frac{\hbar\left(\varkappa_{0}\right)-1}{\mathcal{F}-\mathcal{G} \hbar\left(\varkappa_{0}\right)}\right| \geq 1
$$

which contradicts the fact that $\hbar(\mathcal{x}) \prec \frac{1+\mathcal{F}_{\mathcal{x}}}{1+\mathcal{G}_{\mathcal{X}}}$. Thus $|v(\mathcal{x})|<1$ and so we get the desired proof.

If we replace $g(x)=\frac{\varkappa^{\mathcal{P}+1} \mathrm{q}^{\prime}(x)}{\mathcal{P} \mathrm{q}(x)}$ in (2.1), we acquire the underneath result.

Corollary 2.2. Let $\mathrm{q} \in \AA_{\mathcal{P}}$ and justifying

$$
1+\frac{\alpha \varkappa \mathrm{q}^{\prime}(\varkappa)}{\mathcal{P}^{2} \mathrm{q}(\varkappa)}\left(\mathcal{P}+1+\frac{\varkappa \mathrm{q}^{\prime \prime}(\varkappa)}{\mathrm{q}^{\prime}}-\frac{\varkappa \mathrm{q}^{\prime}(\varkappa)}{\mathrm{q}(\varkappa)}\right)<\frac{1+\mathcal{F}_{\mathcal{X}}}{1+\mathcal{G}_{\varkappa}}
$$

with the condition on $\alpha$ is

$$
|\alpha| \geq \frac{(\mathcal{F}-\mathcal{G}) \mathcal{P}}{(\sin (1)-|\mathrm{B}| \sin \hbar(1))-(1+|\mathrm{B}|) \mathcal{P} \cos \hbar(1)}
$$

Then $\mathrm{q} \in \mathrm{S}_{\mathrm{L}_{\mathcal{P}}}^{*}$.

Theorem 2.3. Let $g \in \AA_{\mathcal{P}}$

$$
1+\frac{\alpha \varkappa g^{\prime}(\varkappa)}{\mathcal{P} g(\varkappa)}<\frac{1+\mathcal{F}_{\varkappa}}{1+\mathcal{G}_{\varkappa}}
$$

With

$$
|\alpha| \geq \frac{(\mathcal{F}-\mathcal{G}) \mathcal{P} \cos \hbar(1)}{(\sin (1)-|\mathrm{B}| \sin \hbar(1))-(1+|\mathrm{B}|) \mathcal{P} \cos \hbar(1)}
$$

Then

$$
\frac{g(x)}{x^{\mathcal{P}}}<\cos \hbar(x)
$$

Proof. let us dene a function 


$$
\hbar(x)=1+\frac{\alpha x g^{\prime}(x)}{\mathcal{P g}(x)}
$$

Then the function $\hbar$ is holomorphic in $\mathfrak{C}$ with $\hbar(0)=1$. Inserting (2.4), we get

$$
\hbar(x)=1+\alpha \frac{x v^{\prime}(x) \sin \hbar(v(x))}{\mathcal{P} \cos \hbar v(x)}+\alpha
$$

and so

$$
\begin{aligned}
\left|\frac{\hbar(x)-1}{\mathcal{F}-\mathcal{G} \hbar(x)}\right|= & \left|\frac{\frac{\alpha \varkappa v^{\prime}(x) \sin \hbar v(x)}{\mathcal{P} \cos \hbar v(x)}+\alpha}{\mathcal{F}-\mathcal{G}\left(1+\frac{\alpha \varkappa v^{\prime}(x) \sin \hbar v(x)}{\mathcal{P} \cos \hbar v(x)}+\alpha\right)}\right| \\
& =\left|\frac{\alpha\left(x v^{\prime}(x) \sin \hbar v(x)+\operatorname{pcos} \hbar v(x)\right)}{(\mathcal{F}-\mathcal{G}) \mathcal{P} \cos \hbar v(x)-\alpha \mathcal{G}\left(x v^{\prime}(x) \sin \hbar v(x)+\mathcal{P} \cos \hbar v(x)\right)}\right|
\end{aligned}
$$

By virtue of Lemma 1.1 along with (2.7) and (2.6), we have

$$
\begin{aligned}
& \left|\frac{\hbar\left(\varkappa_{0}\right)-1}{\mathcal{F}-\mathcal{G} \hbar\left(\varkappa_{0}\right)}\right|=\left|\frac{\alpha\left(\varkappa_{0} v^{\prime}\left(\varkappa_{0}\right) \sin \hbar v\left(\varkappa_{0}\right)+\mathcal{P} \operatorname{cas} \hbar v\left(\varkappa_{0}\right)\right)}{(\mathcal{F}-\mathcal{G}) \mathcal{P} \cos \hbar v\left(\varkappa_{0}\right)-\alpha \mathcal{G}\left(\varkappa_{0} v^{\prime}\left(\varkappa_{0}\right) \sin \hbar v\left(\varkappa_{0}\right)+\mathcal{P} \cos \hbar v\left(\varkappa_{0}\right)\right)}\right| \\
& =\left|\frac{\alpha\left(\mathrm{m} \sin \hbar\left(\mathrm{e}^{\mathrm{i} \theta}\right)+\mathcal{P} \cos \hbar\left(\mathrm{e}^{\mathrm{i} \theta}\right)\right)}{(\mathcal{F}-\mathcal{G}) \mathcal{P} \cos \hbar\left(\mathrm{e}^{\mathrm{i} \theta}\right)-\alpha \mathcal{G}\left(\mathrm{m} \sin \hbar\left(\mathrm{e}^{\mathrm{i} \theta}\right)+\mathcal{P} \cos \hbar\left(\mathrm{e}^{\mathrm{i} \theta}\right)\right)}\right| \\
& \geq\left|\frac{|\alpha|\left(\mathrm{m}\left|\sin \hbar\left(\mathrm{e}^{\mathrm{i} \theta}\right)\right|-\mathrm{p}\left|\cos \hbar\left(\mathrm{e}^{\mathrm{i} \theta}\right)\right|\right)}{(\mathcal{F}-\mathcal{G}) \mathcal{P}\left|\cos \hbar\left(\mathrm{e}^{\mathrm{i} \theta}\right)\right|+|\alpha||\mathcal{G}|\left(\mathrm{m}\left|\sin \hbar\left(\mathrm{e}^{\mathrm{i} \theta}\right)\right|+\mathcal{P}\left|\cos \hbar\left(\mathrm{e}^{\mathrm{i} \theta}\right)\right|\right)}\right| \\
& \geq \frac{|\alpha|(\operatorname{msin}(1)-\mathcal{P} \cos \hbar(1))}{(\mathcal{F}-\mathcal{G}) \mathcal{P} \cos \hbar(1)+|\alpha||\mathcal{G}|(m \sin \hbar(1)+\mathcal{P} \operatorname{cas} \hbar(1))}
\end{aligned}
$$

Now, let

$$
\Xi_{1}(\mathrm{~m})=\frac{|\alpha|(m \sin (1)-\mathcal{P} \cos \hbar(1))}{(\mathcal{F}-\mathcal{G}) \mathcal{P} \cos \hbar(1)+|\alpha||\mathcal{G}|(m \sin \hbar(1)+\mathcal{P} \cos \hbar(1))}
$$

Then

$$
\Xi_{1}^{\prime}(\mathrm{m})=\frac{|\alpha|(\mathcal{F}-\mathcal{G}) \mathcal{P} \cos \hbar(1) \sin (1)+|\alpha|^{2}|\mathcal{G}| \mathcal{P} \cos \hbar(1)(\sin (1)+\sin \hbar(1))}{\{(\mathcal{F}-\mathcal{G}) \mathcal{P} \cos \hbar(1)+|\alpha||\mathcal{G}|(\operatorname{msin} \hbar(1)+\mathcal{P} \cos \hbar(1))\}^{2}}>0
$$

Which illustrates that the function $\Xi_{1}(\mathrm{~m})$ is increasing and hence $\Xi_{1}(\mathrm{~m}) \geq \Xi_{1}(1)$ for $\mathrm{m} \geq 1$

So

$$
\left|\frac{\hbar\left(\varkappa_{0}\right)-1}{\mathcal{F}-\mathcal{G} \hbar\left(\varkappa_{0}\right)}\right| \geq \frac{|\alpha|(\sin (1)-\mathcal{P} \cos \hbar(1))}{(\mathcal{F}-\mathcal{G}) \mathcal{P} \cos \hbar(1)+|\alpha||\mathcal{G}|(\sin \hbar(1)+\mathcal{P} \cos \hbar(1))}
$$

Applying (2.10), we have 


$$
\left|\frac{\hbar\left(\varkappa_{0}\right)-1}{\mathcal{F}-\mathcal{G} \hbar\left(\varkappa_{0}\right)}\right| \geq 1
$$

A contradicts (2.9), this $|v(\varkappa)|<1$ and so the desired proof is completed.

If we replace $g(x)=\frac{\varkappa^{\mathcal{P}+1} \mathrm{q}^{\prime}(x)}{\mathcal{P} \mathrm{q}(x)}$ in last theorem, we obtain the below corollary

Corollary 2.4. Let $q \in \AA_{p}$ and justifying

$$
1+\frac{\alpha}{\mathcal{P}}\left(\mathcal{P}+1+\frac{\varkappa \mathrm{q}^{\prime \prime}(\mathcal{x})}{\mathrm{q}^{\prime}(\mathcal{X})}-\frac{\varkappa \mathrm{q}^{\prime}(\mathcal{x})}{\mathrm{q}(\mathcal{X})}\right)<\frac{1+\mathcal{F}_{\mathcal{X}}}{1+\mathcal{G}_{\varkappa}}
$$

with

$$
|\alpha| \geq \frac{(\mathcal{F}-\mathcal{G}) \mathcal{P} \cos \hbar(1)}{(\sin (1)-|\mathrm{B}| \sin \hbar(1))-(1+|\mathrm{B}|) \mathcal{P} \cos \hbar(1)}
$$

Then $\mathrm{q} \in \mathcal{S}_{\mathrm{L}_{\mathcal{P}}}^{*}$.

Theorem 2.5. Let $g \in \AA_{\mathcal{P}}$ and satisfy the subordination relation

$$
1+\frac{\alpha \mathcal{\varkappa}^{1-\mathcal{P}} g^{\prime}(x)}{\mathcal{P}(g(x))^{2}}<\frac{1+\mathcal{F}_{\varkappa}}{1+\mathcal{G}_{\varkappa}}
$$

with the condition on $\alpha$

$$
|\alpha| \geq \frac{(\mathcal{F}-\mathcal{G}) \mathcal{P}(\cos \hbar(1))^{2}}{(\sin (1)-|\mathrm{B}| \sin \hbar(1))-(1+|\mathrm{B}|) \mathcal{P} \cos \hbar(1)}
$$

is true, then

$$
\frac{g(x)}{\mathcal{x}^{\mathcal{P}}}<\cos \hbar(\varkappa)
$$

Proof. let us dene a function

$$
\hbar(x)=1+\frac{\alpha \varkappa^{1-\mathcal{P}} g^{\prime}(x)}{\mathcal{P}(g(x))^{2}}
$$

Then the function $\hbar$ is holomorphic in $\mathfrak{E}$ with $\hbar(0)=1$. Applying some straightforward calculation, we acquire

$$
\hbar(x)=1+\frac{\alpha x v^{\prime}(x) \sin \hbar(v(x))}{\mathcal{P}(\cos \hbar v(x))^{2}}+\frac{\alpha}{\cos \hbar v(x)}
$$

and so 


$$
\begin{aligned}
& \left|\frac{\hbar(x)-1}{\mathcal{F}-\mathcal{G} \hbar(x)}\right|=\left|\frac{\frac{\alpha \varkappa v^{\prime}(\mathcal{x}) \sin \hbar v(x)}{\mathcal{P}(\cos \hbar v(x))^{2}}+\frac{\alpha}{\cos \hbar v(x)}}{\mathcal{F}-\mathcal{G}\left(1+\frac{\alpha \varkappa v^{\prime}(x) \sin \hbar v(x)}{\mathcal{P}(\cos \hbar v(x))^{2}}+\frac{\alpha}{\cos \hbar v(x)}\right)}\right|
\end{aligned}
$$

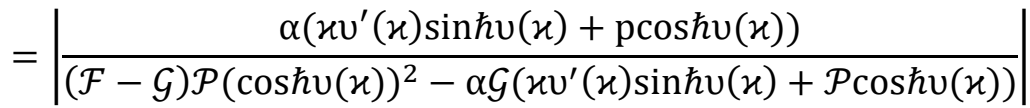

By virtue of Lemma 1.1 along with (2.7) and (2.6), we have

$$
\begin{gathered}
\left|\frac{\hbar\left(\varkappa_{0}\right)-1}{\mathcal{F}-\mathcal{G} \hbar\left(\varkappa_{0}\right)}\right|=\left|\frac{\alpha\left(\varkappa_{0} \mathrm{v}^{\prime}\left(\varkappa_{0}\right) \sin \hbar v\left(\varkappa_{0}\right)+\mathcal{P} \cos \hbar v\left(\varkappa_{0}\right)\right)}{(\mathcal{F}-\mathcal{G}) \mathcal{P}\left(\cos \hbar v\left(\varkappa_{0}\right)\right)^{2}-\alpha \mathcal{G}\left(\varkappa_{0} \mathrm{v}^{\prime}\left(\mathcal{\varkappa}_{0}\right) \sin \hbar v\left(\mathcal{\varkappa}_{0}\right)+\mathcal{P} \cos \hbar v\left(\mathcal{\varkappa}_{0}\right)\right)}\right| \\
=\left|\frac{\alpha\left(\mathrm{m} \sin \hbar\left(\mathrm{e}^{\mathrm{i} \theta}\right)+\mathrm{p} \cos \hbar\left(\mathrm{e}^{\mathrm{i} \theta}\right)\right)}{(\mathcal{F}-\mathcal{G}) \mathcal{P}\left(\cos \hbar\left(\mathrm{e}^{\mathrm{i} \theta}\right)\right)^{2}-\alpha \mathcal{G}\left(\mathrm{m} \sin \hbar\left(\mathrm{e}^{\mathrm{i} \theta}\right)+\mathcal{P} \cos \hbar\left(\mathrm{e}^{\mathrm{i} \theta}\right)\right)}\right| \\
\geq\left|\frac{|\alpha|\left(\mathrm{m}\left|\sin \hbar\left(\mathrm{e}^{\mathrm{i} \theta}\right)\right|-\mathcal{P}\left|\cos \hbar\left(\mathrm{e}^{\mathrm{i} \theta}\right)\right|\right)}{(\mathcal{F}-\mathcal{G}) \mathcal{P}\left(\left|\cos \hbar\left(\mathrm{e}^{\mathrm{i} \theta}\right)\right|\right)^{2}+|\alpha||\mathcal{G}|\left(\mathrm{m}\left|\sin \hbar\left(\mathrm{e}^{\mathrm{i} \theta}\right)\right|+\mathcal{P}\left|\cos \hbar\left(\mathrm{e}^{\mathrm{i} \theta}\right)\right|\right)}\right| \\
\geq \frac{|\alpha|(\mathrm{m} \sin (1)-\mathcal{P} \cos \hbar(1))}{(\mathcal{F}-\mathcal{G}) \mathcal{P}(\cos \hbar(1))^{2}+|\alpha||\mathcal{G}|(\mathrm{m}|\sin \hbar(1)|+\mathcal{P}|\operatorname{cas} \hbar(1)|)}
\end{gathered}
$$

Now, let

$$
\Xi_{2}(\mathrm{~m})=\frac{|\alpha|(\operatorname{msin}(1)-\mathcal{P} \cos \hbar(1))}{(\mathcal{F}-\mathcal{G}) \mathcal{P}(\cos \hbar(1))^{2}+|\alpha||\mathcal{G}|(m \sin \hbar(1)+\mathcal{P} \cos \hbar(1))}
$$

Then

$$
\Xi_{2}^{\prime}(\mathrm{m})=\frac{|\alpha|(\mathcal{F}-\mathcal{G}) \mathcal{P}(\cos \hbar(1))^{2} \sin (1)+|\alpha|^{2}|\mathcal{G}| \mathcal{P} \cos \hbar(1)(\sin (1)+\sin \hbar(1))}{\left\{(\mathcal{F}-\mathcal{G}) \mathcal{P}(\cos \hbar(1))^{2}+|\alpha||\mathcal{G}|(\operatorname{msin} \hbar(1)+\mathcal{P} \cos \hbar(1))\right\}^{2}}>0
$$

Which exhibits that the function $\Xi_{2}(\mathrm{~m})$ is increasing and $\Xi_{2}(\mathrm{~m}) \geq \Xi_{2}(1)$ for $\mathrm{m} \geq 1$, hence

$$
\left|\frac{\hbar\left(\varkappa_{0}\right)-1}{\mathcal{F}-\mathcal{G} \hbar\left(\varkappa_{0}\right)}\right| \geq \frac{|\alpha| \sin (1)-\mathcal{P} \cos \hbar(1)}{(\mathcal{F}-\mathcal{G}) \mathcal{P}(\cos \hbar(1))^{2}+|\alpha||\mathcal{G}|(\operatorname{msin} \hbar(1)+\mathcal{P} \cos \hbar(1))}
$$

Applying (2.13), we have easily achieved

$$
\left|\frac{\hbar\left(\varkappa_{0}\right)-1}{\mathcal{F}-\mathcal{G} \hbar\left(\varkappa_{0}\right)}\right| \geq 1
$$

A contradicts (2.12), this $|v(x)|<1$ and so the desired proof is completed.

If we set $g(x)=\frac{\mathcal{x}^{\mathcal{P}+1} \mathrm{q}^{\prime}(x)}{\mathcal{P} \mathrm{q}(\mathcal{x})}$ in last theorem, we easily have the following corollary.

Corollary 2.6. Let $\mathrm{q} \in \AA_{\mathcal{P}}$ and justifying 


$$
1+\frac{\alpha \mathrm{q}(\mathcal{x})}{\varkappa^{2 \mathcal{P}+1} \mathrm{q}^{\prime}(\varkappa)}\left(\mathcal{P}+1+\frac{\varkappa \mathrm{q}^{\prime \prime}(\varkappa)}{\mathrm{q}^{\prime}(\varkappa)}-\frac{\varkappa \mathrm{q}^{\prime}(\varkappa)}{\mathrm{q}(\varkappa)}\right)<\frac{1+\mathcal{F}_{\mathcal{X}}}{1+\mathcal{G}_{\varkappa}}
$$

With

$$
|\alpha| \geq \frac{(\mathcal{F}-\mathcal{G}) \mathcal{P}(\cos \hbar(1))^{2}}{(\sin (1)-|\mathrm{B}| \sin \hbar(1))-(1+|\mathrm{B}|) \mathcal{P} \cos \hbar(1)}
$$

Then $\mathrm{q} \in \mathcal{S}_{\mathrm{L}_{\mathcal{P}}}^{*}$.

Theorem 2.7. Let $g \in \AA_{\mathcal{P}}$ and satisfy the subordination relation

$$
1+\frac{\alpha \varkappa^{1-2 \mathcal{P}} g^{\prime}(\varkappa)}{\mathcal{P}(g(\varkappa))^{3}}<\frac{1+\mathcal{F}_{\varkappa}}{1+\mathcal{G}_{\varkappa}}
$$

With

$$
|\alpha| \geq \frac{(\mathcal{F}-\mathcal{G}) \mathcal{P}(\cos \hbar(1))^{3}}{(\sin (1)-|\mathrm{B}| \sin \hbar(1))-(1+|\mathrm{B}|) \mathcal{P} \cos \hbar(1)}
$$

Then

$$
\frac{g(x)}{\mathcal{X}^{\mathcal{P}}}<\cos \hbar(x)
$$

Proof. let us dene a function

$$
\hbar(x)=1+\frac{\alpha \mathcal{x}^{1-2 \mathcal{P}} g^{\prime}(\mathcal{x})}{\mathcal{P}(g(\mathcal{H}))^{3}}
$$

So, the function $\hbar$ is holomorphic in $\mathfrak{C}$ with $\hbar(0)=1$. Applying some straightforward calculation, we get

$$
\hbar(x)=1+\frac{\alpha \varkappa v^{\prime}(x) \sin \hbar(v(x))}{\mathcal{P}(\cos \hbar v(x))^{3}}+\frac{\alpha}{(\cos \hbar v(x))^{2}}
$$

and so

$$
\begin{aligned}
\left|\frac{\hbar(x)-1}{\mathcal{F}-\mathcal{G} \hbar(x)}\right|= & \left|\frac{\frac{\alpha x v^{\prime}(x) \sin \hbar v(x)}{\mathcal{P}(\cos \hbar v(x))^{3}}+\frac{\alpha}{(\cos \hbar v(x))^{2}}}{\mathcal{F}-\mathcal{G}\left(1+\frac{\alpha \varkappa v^{\prime}(x) \sin \hbar v(x)}{\mathcal{P}(\cos \hbar v(x))^{3}}+\frac{\alpha}{(\cos \hbar v(x))^{2}}\right)}\right| \\
& =\left|\frac{\alpha\left(x v^{\prime}(x) \sin \hbar v(x)+\mathcal{P} \cos \hbar v(x)\right)}{(\mathcal{F}-\mathcal{G}) \mathcal{P}(\cos \hbar v(x))^{3}-\alpha \mathcal{G}\left(x v^{\prime}(x) \sin \hbar v(x)+\mathcal{P} \cos \hbar v(x)\right)}\right|
\end{aligned}
$$

By virtue of Lemma 1.1 along with (2.7) and (2.6), we have

$$
\begin{aligned}
\left|\frac{\hbar\left(\varkappa_{0}\right)-1}{\mathcal{F}-\mathcal{G} \hbar\left(\varkappa_{0}\right)}\right| & =\left|\frac{\alpha\left(\varkappa_{0} v^{\prime}\left(\varkappa_{0}\right) \sin \hbar v\left(\varkappa_{0}\right)+\mathcal{P} \cos \hbar v\left(\varkappa_{0}\right)\right)}{(\mathcal{F}-\mathcal{G}) \mathcal{P}\left(\cos \hbar v\left(\varkappa_{0}\right)\right)^{3}-\alpha \mathcal{G}\left(\varkappa_{0} v^{\prime}\left(\varkappa_{0}\right) \sin \hbar v\left(\varkappa_{0}\right)+\mathcal{P} \cos \hbar v\left(\varkappa_{0}\right)\right)}\right| \\
= & \frac{\alpha\left(\mathrm{m} \sin \hbar\left(\mathrm{e}^{\mathrm{i} \theta}\right)+\operatorname{pcos} \hbar\left(\mathrm{e}^{\mathrm{i} \theta}\right)\right)}{(\mathcal{F}-\mathcal{G}) \mathcal{P}\left(\cos \hbar\left(\mathrm{e}^{\mathrm{i} \theta}\right)\right)^{3}-\alpha \mathcal{G}\left(\mathrm{m} \sin \hbar\left(\mathrm{e}^{\mathrm{i} \theta}\right)+\mathcal{P} \cos \hbar\left(\mathrm{e}^{\mathrm{i} \theta}\right)\right)} \mid
\end{aligned}
$$




$$
\begin{gathered}
\geq\left|\frac{|\alpha|\left(\mathrm{m}\left|\sin \hbar\left(\mathrm{e}^{\mathrm{i} \theta}\right)\right|-\mathcal{P}\left|\cos \hbar\left(\mathrm{e}^{\mathrm{i} \theta}\right)\right|\right)}{(\mathcal{F}-\mathcal{G}) \mathcal{P}\left(\left|\cos \hbar\left(\mathrm{e}^{\mathrm{i} \theta}\right)\right|\right)^{3}+|\alpha||\mathcal{G}|\left(\mathrm{m}\left|\sin \hbar\left(\mathrm{e}^{\mathrm{i} \theta}\right)\right|+\mathcal{P}\left|\cos \hbar\left(\mathrm{e}^{\mathrm{i} \theta}\right)\right|\right)}\right| \\
\quad \geq \frac{|\alpha|(\mathrm{m} \sin (1)-\mathcal{P} \cos \hbar(1))}{(\mathcal{F}-\mathcal{G}) \mathcal{P}(\cos \hbar(1))^{3}+|\alpha||\mathcal{G}|(\mathrm{m}|\sin \hbar(1)|+\mathcal{P}|\operatorname{cas} \hbar(1)|)}
\end{gathered}
$$

Now let,

$$
\Xi_{3}(\mathrm{~m})=\frac{|\alpha|(\mathrm{m} \sin (1)-\mathcal{P} \cos \hbar(1))}{(\mathcal{F}-\mathcal{G}) \mathcal{P}(\cos \hbar(1))^{3}+|\alpha||\mathcal{G}|(m \sin \hbar(1)+\mathcal{P} \cos \hbar(1))}
$$

Then

$$
\Xi_{3}^{\prime}(\mathrm{m})=\frac{|\alpha|(\mathcal{F}-\mathcal{G}) \mathcal{P}(\cos \hbar(1))^{3} \sin (1)+|\alpha|^{2}|\mathcal{G}| \mathcal{P} \cos \hbar(1)(\sin (1)+\sin \hbar(1))}{\left\{(\mathcal{F}-\mathcal{G}) \mathcal{P}(\cos \hbar(1))^{3}+|\alpha||\mathcal{G}|(\operatorname{msin}(1)+\mathcal{P} \cos \hbar(1))\right\}^{2}}>0
$$

Which exhibits that the function $\Xi_{3}(\mathrm{~m})$ is increasing and $\Xi_{3}(\mathrm{~m}) \geq \Xi_{3}(1)$ from $\mathrm{m} \geq 1$, hence

$$
\left|\frac{\hbar\left(\varkappa_{0}\right)-1}{\mathcal{F}-\mathcal{G} \hbar\left(\varkappa_{0}\right)}\right| \geq \frac{|\alpha| \sin (1)-\mathcal{P} \cos \hbar(1)}{(\mathcal{F}-\mathcal{G}) \mathcal{P}(\cos \hbar(1))^{3}+|\alpha||\mathcal{G}|(\sin (1)+\mathcal{P} \cos \hbar(1))}
$$

Applying (2.16), we have

$$
\left|\frac{\hbar\left(\varkappa_{0}\right)-1}{\mathcal{F}-\mathcal{G} \hbar\left(\varkappa_{0}\right)}\right| \geq 1
$$

A contradicts (2.15), this $|v(x)|<1$ and so the desired proof is completed.

If we set $g(x)=\frac{\mathcal{x}^{\mathcal{P}+1} \mathrm{q}^{\prime}(x)}{\mathcal{P} \mathrm{q}(\mathcal{x})}$ in last theorem, we easily have the following corollary.

Corollary 2.8. Let $\mathrm{q} \in \AA_{\mathcal{P}}$ and justifying

$$
1+\alpha \frac{\mathcal{P}(\mathrm{q}(\mathcal{x}))^{2}}{\mathcal{X}^{3 \mathcal{P}+2}\left(\mathrm{q}^{\prime}(\mathcal{X})\right)^{2}}\left(\mathcal{P}+1+\frac{\varkappa \mathrm{q}^{\prime \prime}(\varkappa)}{\mathrm{q}^{\prime}(\mathcal{X})}-\frac{\varkappa \mathrm{q}^{\prime}(\varkappa)}{\mathrm{q}(\mathcal{X})}\right)<\frac{1+\mathcal{F}_{\mathcal{\varkappa}}}{1+\mathcal{G}_{\varkappa}}
$$

With

$$
|\alpha| \geq \frac{(\mathcal{F}-\mathcal{G}) \mathcal{P}(\cos \hbar(1))^{3}}{(\sin (1)-|\mathrm{B}| \sin \hbar(1))-(1+|\mathrm{B}|) \mathcal{P} \cos \hbar(1)}
$$

Then

\section{Conclusions.}

In the current exploration paper, we inspected a few fascinating traits of starlike functions related with the cosine hyperbolic function. We decide conditions on to such an extent that

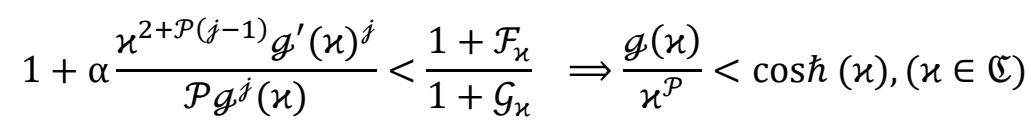


are subordinated by janowski function. Further, choosing some specie values of functions these results are utilized to prove that the function $g$, we get some sufficient criteria for multivalent starlike functions connected with cosine hyperbolic.

\section{References}

[1] Ali, R. M., Cho, N. E., Ravichandran, V., \& Kumar, S. S. (2012). Differential subordination for functions for functions associated with lemniscate of Bernoulli's. Taiwanese journal of mathematics, 16(3): 10171026.

[2] Arif, M., Ahmad, K., Liu, J. L., Sokol, J. (2019): A new class of analytic functions associated with Salagean operator. Journal of Function Spaces, Volume 2019, Article ID 5157394, 8 pages.

[3] Cho, N.E., Kumar, V., Kumar, S.S., \& Ravichandran. V. (2019): Radius problems for starlike functions associated with the sine function. Bulletin of the Iranian Mathematical Society, 45(1) (2019): 213-232.

[4] Cho, N. E., Kumar, S., Kumar, V., Ravichandran, V., Srivastava, H. M. Starlike functions related to the Bell numbers. Symmetry, 11(2) (2019): 219; 17 pages. doi:10.3390/sym11020219.

[5] Dziok, J., Raina, R. K., Sok, J. (2013): On a class of starlike functions related to a shell-like curve connected with Fibonacci numbers. Mathematical and Computer Modelling, 57(56): 12031211:

[6] Halim, S. A., \& Omar, R. (2012): Applications of certain functions associated with lemniscte of Bernoulli. Journal of the Indonesian Mathematical Society, 18(2): 9399:

[7] Haq, M., Raza, M., Arif, M., Khan, Q., \& Tang, H, (2019): Q-analogue of di/erential subordinations. Mathematics, 7(8): 724: Doi:10:3390=math7080724:

[8] Hayman, W., H. (1994): Multivalent functions, Second Edition, Cambridge Uni. Press.

[9] Jack, I. S. (1971): Functions starlike and convex of order alpha. Journal of the London Mathematical Society, 2(3): 469474:

[10] Janowski, W. (1970): Extremal problems for a family of functions with positive real part and for some related families. Annales Polonici Mathematici. 23(2): 159177:

[11] Kanas, S., R...aducanu, D. (2014): Some class of analytic functions related to conic domains. Mathematica Slovaca, 64(5): 11831196:

[12] Kargar, R., Ebadian, A., Sok, J. (2019) On both lemniscate and starlike functions. Complex Analysis and Operator Theory, 9(1): 143154:

[13] Kumar, S., Ravichandran, V., \& Cho, N. E. (2013): Suc cient conditions for starlike functions associated with the lemniscate of Bernoulli. Journal of Inequalities and Applications. 2013(1); Article Id. 176;13 pages.

[14] Kumar, S., Ravichandran, V. (2016): A subclass of starlike functions associated with a rational function. Southeast Asian Bulletin of Mathematics, 40(2): 199212:

[15] Kumar, S., Ravichandran, V. (2018): Subordinations for functions with positive real part. Complex Analysis and Operator Theory. 12(5): 11791191: 
[16] Ma, W., Minda, D. (1994) A unied treatment of some special classes of univalent functions. In Proceedings of the Conference on Complex Analysis; Li, Z., Ren, F., Yang, L., Zhang, S. (Eds), Int. Press: Cambridge, MA, USA, 157169:

[17] Mendiratta, R., Nagpal, S., Ravichandran, V. (2015): On a subclass of strongly starlike functions associated with exponential function. Bulletin of the Malaysian Mathematical Sciences Society, 38(1): 365386:

[18] Paprocki, E., \& Sok, J. (1996): The extremal problem in some subclass of strongly starlike functions. Zeszyty Nauk. Politech. Rzeszowskiej Mat. 20: 8994:

[19] Raina, R. K.; Sok, J. (2015) On coec cient estimates for a certain class of starlike functions. Hacet. J. Math. Stat. 44: 14271433:

[20] Raza, M., Sok, J., \& Mushtaq, S. (2019): Di/erential subordination for analytic functions. Iranian Journal of science and technology, Transactions A: Science. 43(3): 883890:

[21] Sharma, K., Jain, N. K., Ravichandran, V. (2016): Starlike functions associated with a cardioid. Afrika Matematika, 27(56): 923939:

[22] Sharma, K., Ravichandran, V. (2016): Application of subordination theory to starlike functions, Bull. Iran. Math. Soc. 42(3): 761777:

[23] Shi, L., Ali, I., Arif, M., Cho, N. E., Hussain, S., \& Khan, H. (2019): A study of third Hankel determinant problem for certain subfamilies of analytic functions involving cardioid domain. Mathematics, 7(5): 418: Dio:10:3390=math7050418:

[24] Shi, L., Srivastava, H. M., Arif, M., Hussain, S., \& Khan H. (2019): An investigation of the third Hankel determinant problem for certain subfamilies of univalent functions involving the exponential function. Symmetry, 11(5); 598: Dio:10:3390=sym11050598:

[25] Sok, J., Stankiewicz, J. (1996) Radius of convexity of some subclasses of strongly starlike functions. Zeszyty Nauk. Politech. Rzeszowskiej Mat. 19: 101105: 